Ethmane Sall, University of Mulhouse Haute-Alsace, France

DOI:10.17951/Ismll.2020.44.4.91-97

\title{
Le Mali d'autrefois et d'aujourd'hui
}

\author{
Mali - the Past and the Present
}

\begin{abstract}
RÉSUMÉ
Si la fixité territoriale semble érigée en valeur absolue dans le texte de Maryse Condé, le récit de voyage de François-Xavier Freland infirme l'immuabilité des espaces représentés. S'appuyant sur la mécanique intellectuelle des cultures ataviques et des « mythes fondateurs » des empires de l'époque, Condé délègue des personnages qui vantent les vertus de l'immobilité territoriale, considérant que Ségou et Toumbouctou sont éternels. Mais l'écoulement du temps a introduit plusieurs changements dans l'espace malien, à savoir une crise géopolitique provoquée par le chaos libyen et une menace jihadiste prégnante.

Mots-clés : espace malien, immuabilité, idéalisation, mythes fondateurs, flux du temps, métamorphose, déterritorialisation
\end{abstract}

\begin{abstract}
If territorial fixity seems to be erected in absolute value in the text of Maryse Condé, the travel account of François-Xavier Freland invalidates the immutability of the spaces represented. Relying on the intellectual mechanics of "founding myths" of the empires of the time, Condé delegates characters who extol the virtues of territorial immobility, considering that Ségou and Toumbouctou are eternal. But the flow of time has brought several changes to the Malian space, namely a geopolitical crisis brought about by Libyan chaos and a pervasive jihadist threat.

Keywords: Malian space, immuability, idealization, founding myths, flow of time, metamorphosis, deterritorialization
\end{abstract}

\section{Introduction}

Qu'il soit référentiel ou imaginaire, l'espace est fréquemment représenté dans les textes littéraires. Quel que soit le point de vue adopté, il n'est jamais figé : il est mouvant et subit des évolutions au gré du temps. Il perd sa fixité dès lors qu'il est conçu dans la dialectique centrifuge-centripète qui caractérise son articulation : il existerait une tension entre le centrifuge et le centripète, et cette tension encourage la mobilité de l'espace. Dans Mille plateaux, les philosophes Gilles Deleuze et Félix Guattari ont développé une théorie qui met en valeur la complexité de l'espace pour mieux cerner ses enjeux :

Ethmane Sall, Institut de Recherche en Langues et Littératures Européennes, 2 rue des Frères Lumière, 68100 Mulhouse, ethmane.sall@gmail.com, https://orcid.org/0000-0002-3961-7812 
En premier lieu, le territoire lui-même est inséparable de vecteurs de déterritorialisation qui le travaillent du dedans [...]. En second lieu, la D est à son tour inséparable de reterritorialisations corrélatives. C'est que la D n'est jamais simple mais toujours multiple et composée [...]. Or la reterritorialisation comme opération originale n'exprime pas un retour au territoire, mais ces rapports différentiels intérieurs à la D elle-même, cette multiplicité intérieure à la ligne de fuite [...] Au point que la D peut être nommée créatrice de la terre - une nouvelle terre, un univers, et non plus seulement une reterritorialisation (Deleuze \& Guattari, 1980, p. 635).

Cette théorisation du territoire indique à quel point la fixité spatiale est une absurdité. Les deux philosophes distinguent territoire et fixité pour attirer l'attention sur le caractère mouvant de l'espace. L'espace change avec le temps, on ne peut le dissocier des horizons temporels qui déterminent ses « lignes de fuite » (p. 32). Dans les consciences traditionnelles, la conception immuable du territoire est souvent mise en avant pour définir la concentration d'un peuple dans une assise donnée. L'immuabilité du territoire repose sur une forme de « mythe fondateur $»^{1}$ qui légitimerait la présence et la possession du sol par un peuple. Traditionnellement, ce besoin de s'assurer de la propriété exclusive d'un territoire par une communauté est conçu comme une nécessité vitale, nourrie par les dogmes de l'atavisme. Or les histoires des humanités varient en fonction du temps et cette variation détermine les lignes de fuite de l'espace qui est « une totalité diffractée non totalitaire» (Glissant, 2006, p. 72). Par conséquent, il est soumis à « une vitesse infinie de naissance et d'évanouissement » (Deleuze \& Guattari, 1991, p. 112).

Du point de vue « géocritique ( (Westphal, 2007), l'espace est défini comme une fragmentation liée aux lois de la relativité des horizons temporels. Il s'agit d'une poétique qui porterait sur des "interactions entre espaces humains et littérature » (Westphal, 2000, p. 12) et dont l'objet serait de contribuer à « la détermination/ indétermination des identités culturelles » (Westphal, 2000, p. 12). Les liens entre l'espace et le temps sont indissolubles, et on ne peut pas interroger ces liens en dehors de l'altérité : c'est le fondement même de l'articulation de l'approche géocritique de la littérature. La géocritique est une poétique des interfaces qui établit des réseaux de relation entre l'univers fictionnel du texte littéraire et la réalité d'un espace géographique donné. Nous nous fonderons sur ce concept ainsi que sur les travaux d'Édouard Glissant, et de Deleuze et Guattari pour le cadrage théorique de notre propos. Notre contribution portera sur une lecture géocritique de l'espace malien dans Ségou (1984) de Condé et Le Mali, au-delà du jihad (2017) de Freland. Il s'agira de mettre en évidence les bouleversements et les

1 Dans son Introduction à une poétique du divers, Édouard Glissant conceptualise le mythe fondateur de la manière suivante : "Le mythe fondateur rassure obscurément sur la continuité sans faille de cette filiation et autorise dès lors la communauté dont il s'agit à considérer cette terre devenue territoire comme absolument sienne » (Glissant,1996, p. 62). 
métamorphoses de l'espace malien entre le système royal d'autrefois et la terreur jihadiste de l'instant.

\section{Les visages de l'espace malien : entre fixité et relativité territoriales}

Dans le premier tome de Ségou, Maryse Condé décrit l'espace malien d'avant la période de l'expédition coloniale, un espace caractérisé par les imaginaires de l'Afrique traditionnelle. Par glissement de sens, Ségou, capitale du royaume bambara des années 1797, symbolise l'espace malien dans sa complexité. Chef lieu des pouvoirs politiques, culturels et économiques pendant plusieurs siècles, Ségou apparaît comme une zone d'influence d'où s'exerce la force du peuple bambara. La puissance du royaume de Ségou s'étend dans les moindres recoins du territoire malien et parvient à calmer les ardeurs de l'ensemble des forces politiques potentiellement dissidentes : qu'il s'agisse des Dogons, des Somonos, des Bozos, des Sarakolés, des Peuls, des Touaregs, toutes les communautés craignent l'empire royal de Ségou. Face à l'usure du temps qui passe, la romancière guadeloupéenne valorise l'espace de Ségou en le présentant comme un foyer de vie et d'espoir à travers des envolées lyriques qui soulignent son immuabilité. Plus de deux siècles après, " le temps a changé et l'espace a changé » (Glissant, 2006, p. 24) : le Mali d'autrefois est bien différent du Mali d'aujourd'hui, on est passé d'un royaume prééminent à un État-nation déstabilisé. Le récit du journaliste FrançoisXavier Freland en fait foi : il met en scène un espace malien épouvanté par les mouvements jihadistes qui touchent particulièrement le Nord du pays.

Il convient de contextualiser historiquement le propos pour mieux discerner les déterminants politiques de la déterritorialisation de l'espace malien. Le système royal d'autrefois concerne l'histoire précoloniale du Mali : il est caractérisé par l'épopée mandingue de Soundjata Keïta et de son successeur Kankou Moussa à travers l'Empire du Mali $\left(\mathrm{XI}^{\mathrm{e}}-\mathrm{XVI}^{\mathrm{e}}\right.$ siècles$)$, et le royaume Bambara (XVII ${ }^{\mathrm{e}}$ - XIX ${ }^{\mathrm{e}}$ siècles). Le personnage de Da Monzon autour duquel se cristallise l'idéalisation de l'espace malien dans Ségou est l'un des derniers dignitaires du royaume Bambara. L'Empire Toucouleur d'Elhadj Omar Tall a commencé en 1861 après la chute de ce royaume. Une première dose de déterritorialisation se profile déjà dans ces différents mouvements politiques et indique les limites de la fixité territoriale que l'on peut lire dans certains passages de Ségou. Il n'est pas question de faire une étude chronologique de l'histoire malienne. Toutefois, nous tenons à souligner une autre mesure de déterritorialisation qui intervint pendant et après l'expédition coloniale. C'est durant la période de l'Empire d'Elhadj Omar Tall que la France envahit le Mali. Depuis cette période, ce territoire de l'Afrique de l'Ouest connut de nombreuses mutations sociales et politiques. Au-delà de la tempête coloniale, l'une des plus marquantes de ces mutations fut et est la naissance de la rébellion Touareg de Kidal en 1962. Du régime de Modibo Keïta (1960-1968) à la présidence d’Ibrahim Boubacar Keïta (de 2013 
jusqu'à nos jours), cette rébellion s'est progressivement développée et a donné naissance à l'impasse politique à laquelle le pouvoir malien est confronté aujourd'hui.

S'appuyant sur l'imaginaire des cultures ataviques et des «mythes fondateurs », Maryse Condé présente Ségou sous l'angle d'un idéalisme qui révèle la fixité territoriale du royaume bambara. Cet imaginaire consiste à « consacrer la présence d'une communauté sur un territoire, en rattachant par filiation légitime cette présence » (Glissant, 1996, p. 62). En ce sens, il est question d'une inflexibilité de l'espace dont l'axe de temps s'entretient pour se prémunir de la dynamique décomposition/recomposition territoriale à laquelle les espaces humains sont soumis. Ségou serait ainsi un endroit mythique :

Ces singes rouges qui vous terrifient tant seront bientôt défaits jusqu'au dernier par d'autres musulmans, venus ceux-là, du Fouta Toro. Il ne restera rien de la capitale qu'ils vont bâtir sur la rive droite du Bani et à qui dans leur arrogance ils vont donner le nom de leur Dieu. Ils redeviendront éleveurs comme devant. Tandis que, croyez-moi, Ségou est éternelle. Son nom traversera les siècles. Après vous, les enfants de vos enfants le répéteront (Condé, 1984, pp. 161-162).

Face à la colonisation arabo-musulmane, Maryse Condé délègue Soumaoro Bagayoko, féticheur réputé dans la communauté bambara, pour rappeler le caractère «strié » de Ségou. Il utilise la mécanique intellectuelle de l'atavisme territorial pour suivre les trajets coutumiers qui lui permettraient de sauvegarder Ségou, de la maintenir intacte. Or, avec Deleuze et Guattari, il convient de rappeler que l'espace humain « se métamorphose en changeant de nature », il renferme des « lignes de segmentarité, de stratification » (Deleuze \& Guattari, 1980, p. 32) qui le déterritorialisent sans cesse pour le reterritorialiser dans de nouveaux cadres. Le rapport binaire (Ségou et les autres espaces) de la position de Bagayoko présente des limites si l'on tient compte de ce témoignage de Freland :

Mais la tempête est proche, à l'image des articles ouvertement racistes dans la presse de Bamako à l'égard des Touaregs. Les actes de violence se multiplient, parfois gratuitement, dans la capitale malienne $[\ldots]$.

Le Nord du pays donne l'impression de craquer littéralement face aux coups de boutoir de ces rebelles touaregs, certes peu nombreux mais extrêmement efficaces (Freland, 2017, p. 54).

La crise géopolitique de la sous-région qui résulte du chaos libyen a provoqué un bouleversement majeur au Mali. Elle a bousculé les structures politiques, occasionnant un changement sans précédent au sein de l'espace malien. Si Ségou est « éternelle » pour le protagoniste de Condé, elle devient instable avec le récit de Freland. Le cadre décrit par Condé donne l'impression d'un espace sensiblement imaginaire. Or ce cadre 
a existé. Il a donc subi les lois de la variabilité du « devenir $»^{2}$ et celles de la relativité de «l'étant $»^{3}$. Les notions du « devenir » et de « l'étant» s'opposent à l'absolu de l'être, souvent conçu comme une donnée identique à soi-même. Les deux notions renvoient au mouvement et au changement par rapport à la nature humaine et aux espaces qu'elle habite. Elles permettent de mieux cerner la question de la variabilité de l'espace humain. Le cadre malien peint par Maryse Condé s'inscrit dans une conjoncture temporelle bien située dans l'histoire du pays. Cette conjoncture n'est plus. La dimension « éternelle » de Ségou relève d'une vision hypertrophiée. Plus de deux siècles après, Ségou apparaît comme un amas d'atomes, un espace fragmenté qui « met en jeu des êtres d'échelles et des règnes tout à fait différents » (Deleuze \& Guattari, 1980, p. 291) sans aucune forme d'immuabilité possible. Ainsi, la géocritique révèle la « foncière mobilité des espaces humains et des identités culturelles qu'ils véhiculent » (Westphal, 2000, p. 13) : le changement de nature de l'espace conditionne la variabilité de « l'étant» chez les individus qui l'habitent. Le Ségou d'avant et le royaume bambara ne peuvent perdurer dans l'absolu.

\section{La sédentarité de la terre et la mobilité du « lieu »}

En fondant les États-nations, la notion de terre et les identités monolithiques ont donné lieu à plusieurs tensions entre entités nationales. Ces tensions sont alimentées par les dogmes d'une fixation qui cherche à « distribuer aux hommes un espace fermé, en assignant à chacun sa part, et en réglant la communauté des parts » (Deleuze \& Guattari, 1980, p. 472). Le « lieu » ${ }^{4}$ s'articule comme le fondement d'une pensée philosophique qui établit des liaisons entre les espaces humains et l'utopie créatrice du " Tout-Monde ». Chez Édouard Glissant, le « lieu » ne requiert pas forcément une référence à une nation particulière, c'est l'endroit que l'individu se choisit pour définir sa relation au monde. La théorisation du «lieu » par Glissant éclaire la question de la mobilité de l'espace dans la mesure où le poète-philosophe martiniquais abolit toute forme de fixité qui encouragerait le cantonnement des espaces humains.

2 Dans Mille plateaux, Deleuze et Guattari théorisent le devenir ainsi : « Le devenir est toujours d'un autre ordre que celui de la filiation. Il est de l'alliance [...]. Il met en jeu des êtres d'échelles et des règnes tout à fait différents, sans aucune filiation possible » (Deleuze \& Guattari 1980, p. 291).

3 Édouard Glissant fait une nette distinction entre « l'être » et « l'étant » pour mettre en garde contre les dérives de l'identique : « [...] on peut se promener dans l'étant, il comporte des territoires, non des terres, il admet l'étendue, et que s'il est difficile de parler des qualités de l'Être, il est possible de le faire pour l'étant, mais ces qualités de l'étant n'en sont pas, ce sont des variables » (Glissant, 2006, pp. 178-179).

4 Leitmotiv de Glissant, le « lieu », réel ou symbolique, est conceptualisé de la manière suivante : «Le lieu est incontournable [...]. Impossible de dire là où commence et là où s'achève le lieu parce que la frontière est imperméable [...]. Mais si vous désirez profiter dans ce lieu qui vous a été donné, réfléchissez que désormais tous les lieux du monde se rencontrent jusqu'aux espaces sidéraux » (Glissant, 1993, p. 29). 
Chez Condé, c'est la sublimation de l'action politique de l'ancien roi de Ségou qui favoriserait l'idée de la sédentarité territoriale. Cette sublimation rassurerait opiniâtrement sur la question de la pérennité sans faille de l'espace représenté. Par extension de légitimité territoriale, cette continuité est vécue comme «l'harmonie d'une Mesure » (Glissant, 2005, p. 223) :

Depuis son accession au trône, Da Monzon avait apporté nombre de modifications à l'ameublement du palais. Il avait fait construire une sorte de salon particulier avec des fauteuils d'Europe et des canapés très bas couverts de couvertures marocaines [...]. Ainsi la nuit n'existait plus et le souverain avait reçu un nouveau titre qui s'ajoutait à ceux qu'il possédait déjà : maître de la bataille, long serpent protecteur de Ségou, source de vitalité. C'était celui de « maître des soleils de la nuit » (Condé, 1984, p. 134).

La conscience historique de la sédentarité de Ségou repose sur le personnage mythifié de Da Monzon. À travers cette figure mythique, l'espace malien devient davantage terre et genèse plutôt que « lieu » et « relation ». Le « lieu » et la « relation » sont variables, ils emmêlent les histoires et encouragent la mobilité de l'espace. La terre et la genèse sont fixes, elles supposent l'idée d'une « légitimation universelle de la présence [d'une] communauté » (Glissant, 1996, p. 62), donc d'un absolu fondé sur une filiation qui ne consentirait pas au devenir. Là où le « lieu » met en jeu des " régimes de signes différents et même des états non-signes » (Deleuze \& Guattari, 1980, p. 31), la terre tend à sédentariser. Naturellement, le changement du temps entraîne la variation de l'espace. C'est ce qu'on lit dans ce passage du texte de Freland :

La désinvolture qui règne au palais présidentiel de Koulouba face à cette dégradation de la situation militaire dans le Nord est encore plus inquiétante. La situation semble dépasser l'ancien stratège militaire, le général Amadou Toumani Touré en personne, qui donne l'impression de vouloir se détourner d'une situation qu'il a peut-être lui-même laissée se dégrader [...].

Et peu à peu, au Mali, l'indifférence du début se meut en une peur irrationnelle. Cette terre de partage et de cohabitation séculaire se divise dans un repli identitaire (Freland, 2017, pp. 54-55).

Par glissement de sens, le palais de Koulouba et le pouvoir politique d'Amadou Toumani Touré correspondent à ceux de Da Monzon. Il s'agit de correspondances éclatées qui témoignent de l'incroyable bouleversement subi par l'espace malien au fil du temps. Les cadres des « palais » royal et présidentiel sont des métonymies qui représentent le territoire malien à travers deux époques différentes, un territoire secoué profondément par une crise majeure qui semble échapper aux prévisions politiques. Sur le plan géocritique, les deux « palais » entretiennent des relations de transposition et de transfiguration non revendiquées. Ces relations permettent d'évaluer le degré de chamboulement spatial entraîné par les horizons temporels qui séparent les contextes des deux récits. 
Aujourd'hui, le pouvoir en place est confronté à un vent de soulèvement populaire qui a provoqué la démission du Président et un début de renouvellement d'imaginaires politiques. Cette flamme de la résistance populaire, née de l'inaction d’Ibrahim Boubacar Keïta, énonce une autre mesure de déterritorialisation.

\section{References}

Condé, M. (1984). Ségou. Les Murailles de terre. Paris: Robert Laffont.

Deleuze, G., \& Guattari, F. (1980). Mille plateaux. Capitalisme et schizophrénie 2. Paris: Minuit.

Deleuze, G., \& Guattari, F. (1991). Qu'est-ce que la philosophie? Paris: Minuit.

Freland, F.-X. (2017). Mali. Au-delà du jihad. Paris: Anamosa.

Glissant, É. (1993). Tout-Monde. Paris: Gallimard.

Glissant, É.(1996). Introduction à une poétique du divers. Paris: Gallimard.

Glissant, É.(2005). La Cohée du Lamentin. Paris: Gallimard.

Glissant, É.(2006). Une Nouvelle région du monde. Esthétique I. Paris: Gallimard.

Westphal, B. (2000). Pour une approche géocritique des textes. In B. Westphal (2000), La Géocritique mode d'emploi (pp. 9-40). Limoges: Presses universitaires de Limoges. Retrieved March 11, 2020 , from http://sflgc.org/bibliotheque/westphal-bertrand-pour-une-approche-geocritique-des-textes/.

Westphal, B. (2007). La Géocritique. Réel, fiction, espace. Paris: Minuit. 